\title{
Molecular Architecture of Bacterial Flagellar Stator Revealed by Cryo-Electron Tomography
}

\author{
Xiaowei Zhao*, Joshua E. Pitzer**, Md A. Motaleb**, Steven, J. Norris*, Jun Liu*, \\ * Department of Pathology and Laboratory Medicine, University of Texas Medical School at Houston, \\ Texas 77030 \\ ** Department of Microbiology and Immunology, Brody School of Medicine, East Carolina University, \\ Greenville, NC 27834
}

Bacterial flagella are helical propellers turned by the flagellar motor, a remarkable nano-machine embedded in the bacterial cell envelope $[1,2]$. Powered by the proton gradient across the cytoplasmic membrane, the motor converts electrochemical energy into torque through an interaction between the rotor and the stator $[3,4]$. The stator of the flagella motor, composed of the membrane protein complexes (MotA/MotB), functions as a proton channel to couple proton flux with motor rotation and as anchor that stabilizes the motor to cell wall. Mutations in $\operatorname{mot} A$ or $\operatorname{mot} B$ cause a paralyzed flagellum. Direct structural information of the stator and its precise location inside the flagellar motor has so far been limited. Recently, cryo-electron tomography (cryo-ET) of Lyme disease spirochete Borrelia burgdorferi revealed intact flagellar motor structure at $3.5 \mathrm{~nm}$ resolution, yet the stator-rotor interface was not well defined because of the complexity [5]. In this work, a motB mutant was constructed by a newly developed non-polar gene inactivation system and was also complemented successfully (Fig.1a). The non-polar $m o t B$-cells synthesize periplasmic flagella but were paralyzed (Fig. 1b). The defect was corrected when the mutant was complemented $(\operatorname{mot} B+)$ in trans. The flagellar motor structures of wild-type (WT), $\operatorname{mot} B$ mutant $(m o t B-)$, and $m o t B$ complement $(m o t B+)$ were reconstructed and analyzed comparatively. A stator ring composed by sixteen MotA/MotB complexes was structurally determined for the first time. It located peripherally around the rotor as shown in Fig. 2. The stator-rotor interaction provides new insight into the fundamental mechanism of flagellar rotation and bacterial motility.

\section{References}

[1] Berg, H.C. (2003). The rotary motor of bacterial flagella. Annu Rev Biochem 72, 19-54.

[2] Sowa, Y., and Berry, R.M. (2008). Bacterial flagellar motor. Q Rev Biophys 41, 103-132.

[3] Kojima, S., and Blair, D.F. (2001). Conformational change in the stator of the bacterial flagellar motor. Biochemistry 40, 13041-13050.

[4] Kim, E.A., Price-Carter, M., Carlquist, W.C., and Blair, D.F. (2008). Membrane segment organization in the stator complex of the flagellar motor: implications for proton flow and proton-induced conformational change. Biochemistry 47, 11332-11339.

[5] Liu, J., Lin, T., et al (2009). Intact flagellar motor of Borrelia burgdorferi revealed by cryoelectron tomography: evidence for stator ring curvature and rotor/C-ring assembly flexion." J Bacteriol 191(16): 5026-36. 

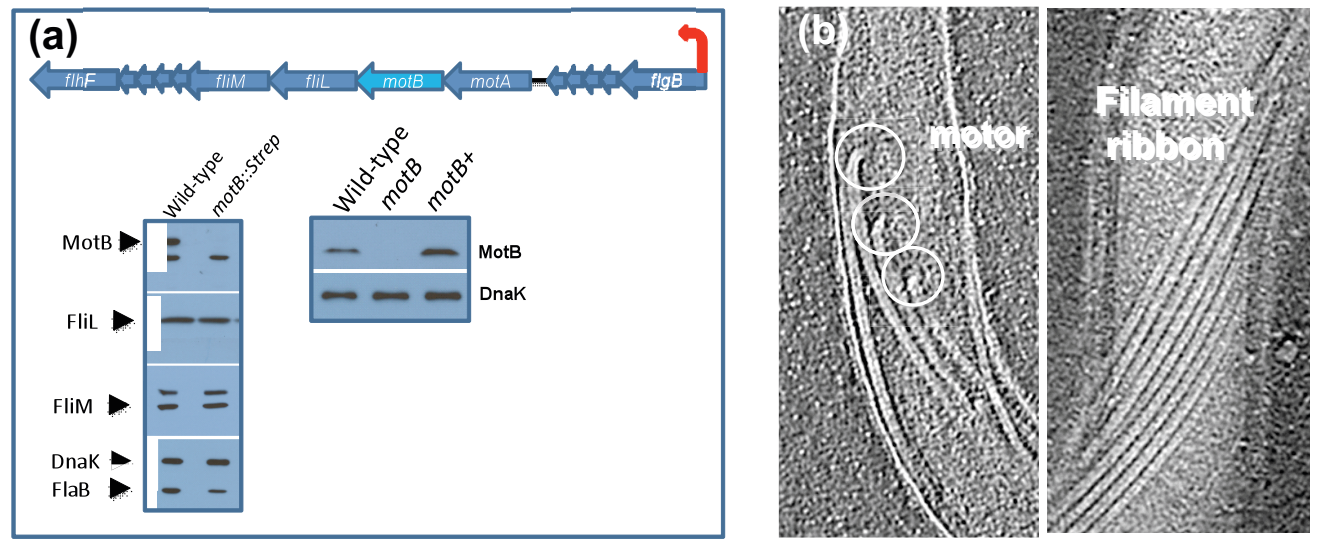

Fig. 1 (a) MotB protein is detected in the WT and the complemented motB + cells but is inhibited in the mutant cells. No polar effect on downstream FliL or FliM synthesis is detected (FliL/FliM blots). Inactivation of $\operatorname{mot} \mathrm{B}$ had a minor affect on FlaB synthesis (FlaB blot). Dnak is used as a loading control. (b) Tomograms of mot $B$ - cells. motB-cells are nonmotile and rod-shaped, but the flagella are assembled as a ribbon.
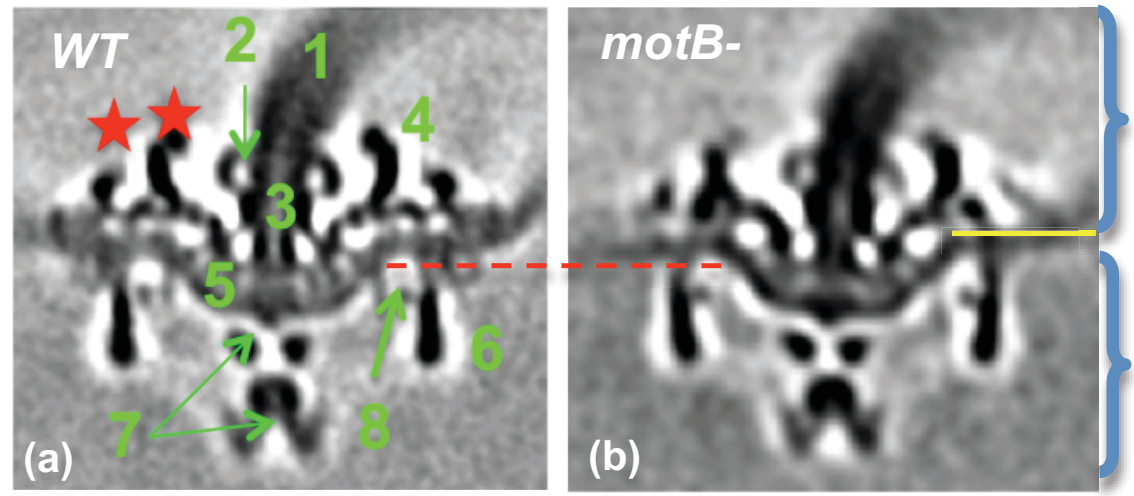

Periplasmic space

membrane
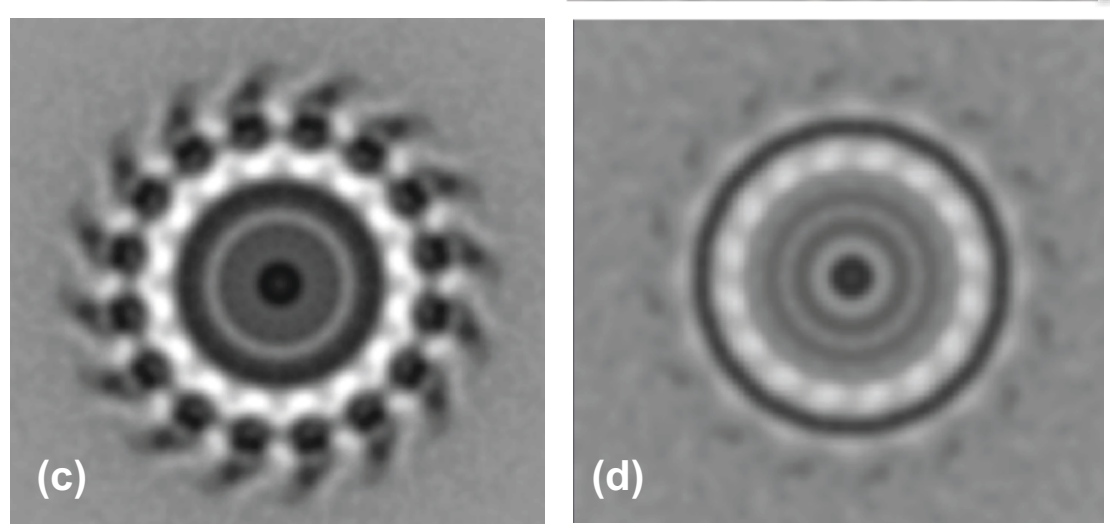

cytoplasmic

space

Fig. 2 Three dimensional density maps averaged WT and motB-motors. (a) and (b): side-view. The numbers in Fig. 2(a) represent: 1. hook, 2. P ring, 3. rod, 4. collar, 5. MS ring, 6. C ring, 7. export apparatus, and 8. FliG. The red stars indicate the "inner collar" (near the hook) and "outer collar". (c) and (d) plane-view of WT and motB- motors showing the slicers indicated by red dashed line. A ring composed by 16 subunits is assumed to be the stator ring in WT motor (c). This ring is not presented in $\operatorname{mot} B$ - motor $(\mathrm{d})$. 